\title{
Meiotic prophase I defects in an oligospermic man with Wolf-Hirschhorn syndrome with ring chromosome 4
}

Qi Yao ${ }^{2+}$, Liu Wang ${ }^{3+}$, Bing $\mathrm{Yao}^{2+}{ }^{+}$, Hongliu Gao ${ }^{1+}$, Weiwei $\mathrm{Li}^{1}$, Xinyi Xia ${ }^{1}$, Qinghua Shi ${ }^{3}$ and Yingxia Cui ${ }^{*}$

\begin{abstract}
Background: Ring chromosomes are often associated with spermatogenetic failure. However, the mechanism is poorly understood. We here reported a single man with severe oligospermia and a ring chromosome 4 with a microdeletion at $4 \mathrm{p} 16.3$.

Results: Synapsis (as SCP3), recombination (as MLH1) and transcriptional inactivation (as BRCA1) in a testicular biopsy were examined by fluorescence immunostaining. In the oligospermia patient, 35.4\% of spermatocytes were in zygotene phase compared with $5.2 \%$ in controls. The patient had a significantly reduced recombination frequency with mean of $45.9 \mathrm{MLH} 1$ foci/cell compared with 47.8 in controls. In the patient, chromosome 4 in all pachytene cells displayed loop formation with varying degrees of unpaired regions. BRCA1 localized along asynapsed regions regardless of XY body association.
\end{abstract}

Conclusions: Ring chromosome 4 might affect the progression of meiosis I prophase, synapse formation, and transcriptional activation of asynapsed areas, and impair male fertility.

Keywords: Ring chromosome 4, Oligospermia, Synapse complex, Recombination, Transcriptional inactivation

\section{Background}

Chromosomal structural abnormalities such as inversion, translocation and complex chromosome rearrangements can disturb the first meiotic division and result in sterility [1-4]. Ring chromosomes are rare chromosomal structural abnormalities. The phenotypes of patients with ring chromosome include physical and mental defects due to loss of genomic material and ring formation [5], as well as spermatogenic arrest [6-16]. However, the underlying mechanisms of spermatogenetic failure in patients with ring chromosomes are not fully understood. Here, we report a patient with ring chromosome 4 and microdeletion of chromosome region 4p16.3 presenting the core features of Wolf-Hirschhorn syndrome and spermatogenic arrest. To understand the mechanisms of spermatogenic arrest in this patient, this study used fluorescence immunocytogenetic methods to investigate

\footnotetext{
* Correspondence: cuiyingxia55@126.com

'Equal contributors

'Institute of Laboratory Medicine, Jinling Hospital, Nanjing University School of Medicine, 305 East Zhongshan Road, Nanjing 210002, PR China Full list of author information is available at the end of the article
}

the progression of meiosis I prophase, chromosome pairing and recombination, transcriptional inactivation.

\section{Case presentation}

\section{Patient}

The patient was a 23-year-old man who presented at our center for oligospermia. The patient showed signs of Wolf-Hirschhorn syndrome. He had left-side cryptorchidism. His right scrotal testis was slightly soft and small (12 mL; normal testes size for adult Chinese $19.8 \pm$ $7.1 \mathrm{~mL}$ ). His penis was appropriate in size. Semen analysis revealed a semen volume of $1 \mathrm{ml}$, sperm concentration of 2.81 million per ml, and motility of $14.63 \%$, with a total motile sperm count of 0.41 million. A repeat sample 2 weeks later showed a semen volume of $1 \mathrm{ml}$, sperm concentration of 0.73 million per $\mathrm{ml}$, and motility of $12 \%$, with a total motile sperm count of 0.09 million. A CT brain scan found no abnormality. Abdominal ultrasound revealed no visceral anomalies. Intelligence was in the lower limit of the normal range. After obtaining informed content from the patient and his parents, biopsy was performed on the right testis. Histology of the testicular 
biopsy sample showed the number of primary spermatocytes was reduced, and spermatogenic arrest at the primary spermatocyte stage (Figure 1).

Hormonal profile was normal for serum concentrations of PRL, LH, and T. FSH concentration was higher than normal (18.9 IU/L, normal value 1.0-5.5 IU/L). Ychromosome screening for AZF microdeletions was normal.

Karyotype analysis of peripheral blood lymphocytes by classic G-binding revealed 46,XY,r(4)(::p16.3 $\rightarrow$ q35.2::) [31]/45,XY,-4[3] (Figure 2A). To characterize the ring chromosome 4, molecular cytogenetic analysis was performed using the Affymetrix Genome-wide Human SNP array 6.0 platform (Affymetrix Inc., Santa Clara, CA). Array data were analyzed using Genotyping Console software. The results indicated a terminal $852 \mathrm{~kb}$ deletion at 4p16.3 that included 17 annotated genes (MIRN571, LOC654254, ABCA11, LOC642707, ZNF141, ATP51, GAK, ZNF595, MGC26356, ZNF718, MFSD7, PDE6B, PCGF3, MYL5, PIGG, ZNF721, and CPLX1) (Figure 2B). Thus, the final karyotype was 46,XY,r(4)(::p16.3 $\rightarrow$ q35.2::)dn.arr[hg18]4pterp16.3(57,770-910,354) × 1[31]/ $45, \mathrm{XY},-4[3]$. Parental karyotypes were normal, indicating de novo origin of the ring chromosome 4.

\section{Materials and methods}

\section{Meiotic analyses}

Testicular tissue was processed as described previously [17]. Control samples C1-C5 were obtained from five prostate cancer patients with proven fertility who had undergone orchiectomy (age range: 64-69 years). Informed consent was obtained from the patient, his parents

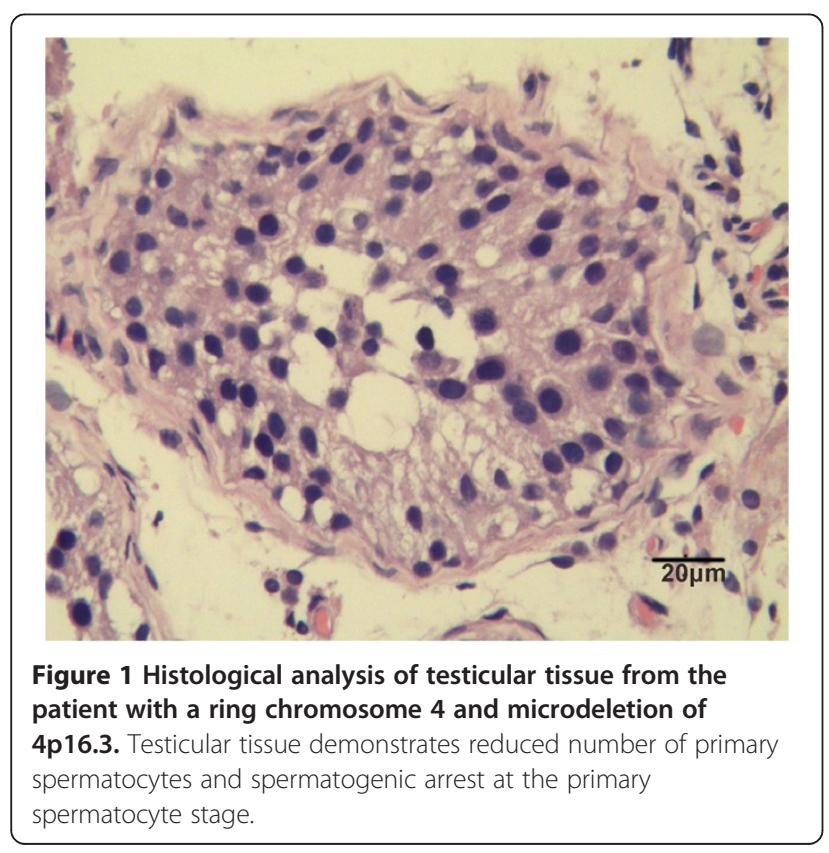

and the five control patients, and research was approved by the Ethics Committee of the Nanjing Jinglin Hospital.

Primary antibodies were rabbit anti-SCP3 (donated by Dr Christa Heyting, University of Wageningen, The Netherlands), human anti-CREST (Immunovision, Springdale, AR), mouse anti-MLH1 (BD Pharmigen Bioscience, San Diego, CA), and mouse anti-BRCA1 (Santa Cruz Biotechnology, Santa Cruz, CA). Primary antibodies were detected using secondary antibodies Alexa 555 donkey anti-rabbit (Molecular Probes, Carlsbad, CA), Alexa 488 goat anti-mouse (Molecular Probes), and 1-amino-4-methylcoumarin-3-acetic acid (AMCA) donkey anti-human (Jackson Immunoresearch, West Grove, PA).

Cell evaluation and image capturing were by epifluorescence microscopy (Olympus BX61, Olympus Inc., Tokyo, Japan) and Image Pro-Plus version 5.1 software (Media Cybernetics Inc., Bethesda, MD). The progression of meiotic prophase I were distinguished by the appearance and chronology of synaptonemal complex (SC) protein SCP3. Multiple short SCP3-positive segments were revealed at leptotene; 46 complete but unpaired SCP3-positive elements with 46 CREST signals (a mark of centromere) were observed at zygotene; synapsis of homologues was completed with 23 CREST, 23 SCs signals and the appearance of MLH1 foci (a mark of meiotic recombination sites) at pachytene.

Statistical analysis used SPSS 16.0 software (SPSS Inc., Chicago, IL). The Mann-Whitney test was used to compare MLH1 foci per cell between the patient and controls. A chi-square test was applied to compare recombination rates of $\mathrm{XY}$ pairs in the patient and controls.

\section{Results}

Meiotic prophase I substages were identified in the patient's biopsy sample using immunofluorescence to determine the appearance and chronology of SCP3. 164 primary spermatocytes from the patient and 1828 from five control men were analyzed. For the five controls, mean frequencies of cells by stage were $13.8 \%$ for leptotene, $5.2 \%$ for zygotene, and $81 \%$ for pachytene. In the patient, $35.4 \%$ of cells were in zygotene stage, indicating blockage at this stage; with $12.2 \%$ of cells in leptotene stage, and $52.4 \%$ in pachytene stage.

For the patient and for each of the five controls, recombination frequencies were determined for 73 pachytene nuclei. In the patient, the mean number of MLH1 foci per cell was significantly lower than the number in controls $(45.9 \pm 5.9$ vs. $47.8 \pm 4.5 P=0.004$, Mann-Whitney test). However, the recombination frequency in the patient, as determined by $\mathrm{XY}$ bivalents, was not significantly different from the controls $(76 \%$ vs. $78 \%, P=0.731$, chi-square test). 


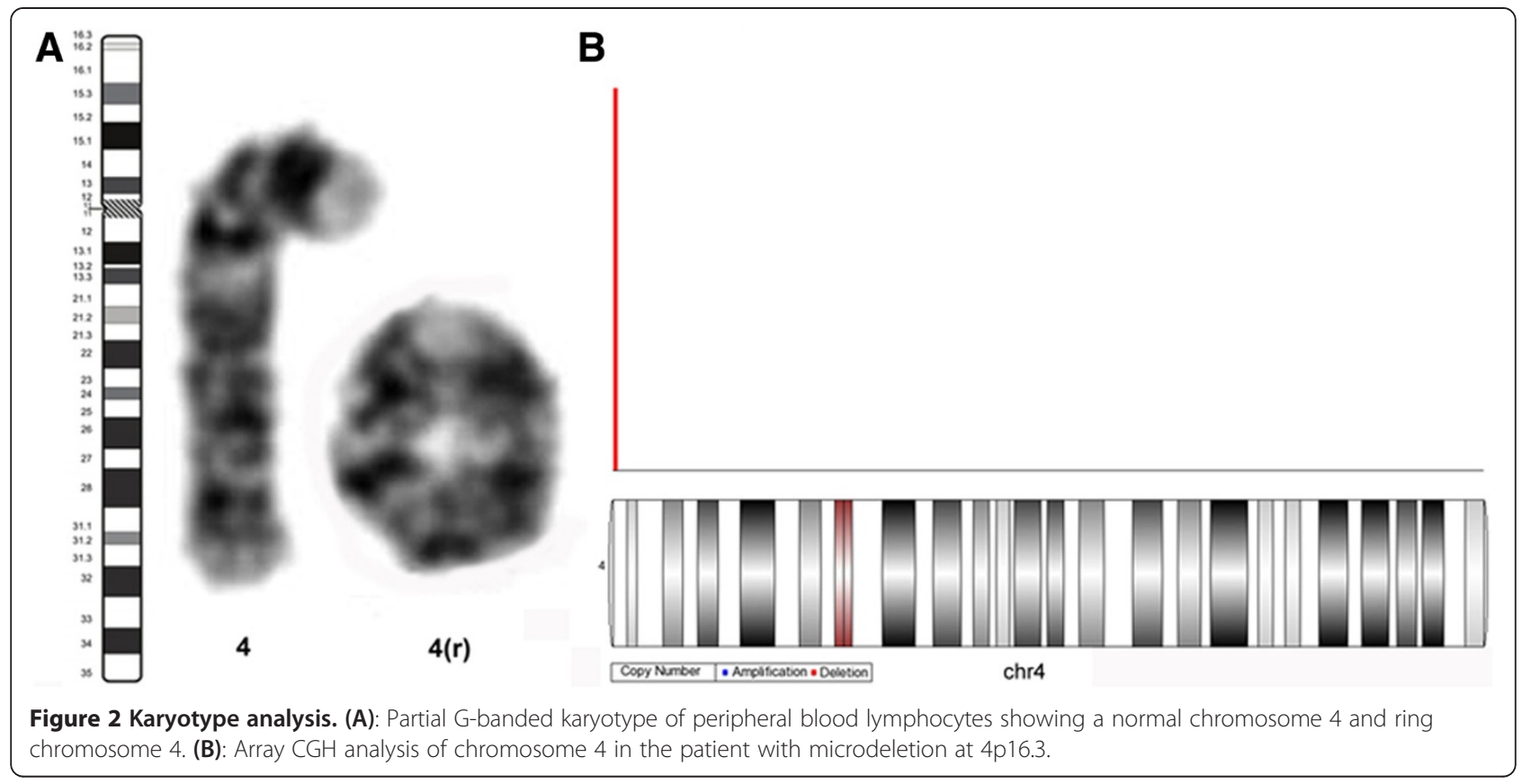

In all pachytene nuclei analyzed from the patient, the chromosome 4 homologs paired and formed loops with two free ends. The loops showed different degrees of asynapsis between the homologs (Figure 3).

In pachytene nuclei of the patient carrying ring chromosome 4, BRCA1 staining was seen in the X and $\mathrm{Y}$ axis (excluding the pseudoautosomal region) and in the asynaptic segments of the axes of chromosome 4 (Figure 3B-C).

\section{Discussion}

Wolf-Hirschhorn syndrome is a congenial anomaly due to a deletion in the distal short arm of chromosome 4 . The main clinical picture is characterized by dysmorphic facial features, delayed growth, delayed psychomotor and multiple malformations [18-20]. The syndrome was often diagnosed at early life. However, in this case, the diagnosis has not been made until adulthood, which let us find a novel phenotype of spermatogenic arrest.

A series of complex processes involving pairing, synapsis, recombination, and segregation of homologous chromosomes take place during the first meiotic division. When any of these processes is altered, cellular checkpoints will arrest the progression of meiosis and induce cell loss, which causes a severe reduction in fertility, or even sterility. The main characters of spermatogenesis in the present patient were synapse defect between the homologs of chromosome 4 and pachytene arrest. The conditions confirmed synapse defect might trigger meiosis checkpoints and lead to spermatogenic arrest at the pachytene stage and finally, resulting in spermatogenesis failure. Three checkpoints are reported to be responsible for meiotic arrest at the zygotene-pachytene transition, including DNA double-strand breaks (DSBs), SC and meiotic inactivation of sex chromosome (MSCI) [21-24]. The operation of all these three checkpoints can be triggered by errors in chromosomes synapsis (also called asynapsis) when asynapsed chromosome segments reach a threshold. As the configuration of loops varied from cell to cell, different checkpoints might be triggered in different spermatocytes even in the same patient. Therefore, for the patient, unpaired fragments in most primary spermatocytes reached the threshold, displaying spermatogenic arrest at the pachytene stage, while unsynapsis segments in a few cells may be too small to be detected by the pachytene checkpoints, revealing a phenotype of oligozoospermia.

In mammals, asynapsis in males is almost always associated with spermatocyte losses during the pachytene stage of meiotic prophase and/or at the metaphase stage of the first meiotic division, with consequent subfertility or sterility [22]. It has been proposed that unsynapsed chromosomal regions could drive a process called meiotic silencing of unsynapsed chromatin (MSUC), which may disrupt the normal loading of MSUC proteins, interfere with autosome and sex chromosome gene expression [21,22] and trigger a massive pachytene cell death. In our case, synapse defect of the homologs of chromosome 4 was linked to reduction of primary spermatocytes in seminiferous tubular. The mechanism of reproductive impairment might be ascribed to MSUC and further lead to pachytene apoptosis.

Compared with control patients, a significant reduction in the mean number of MLH1 foci per cell was 


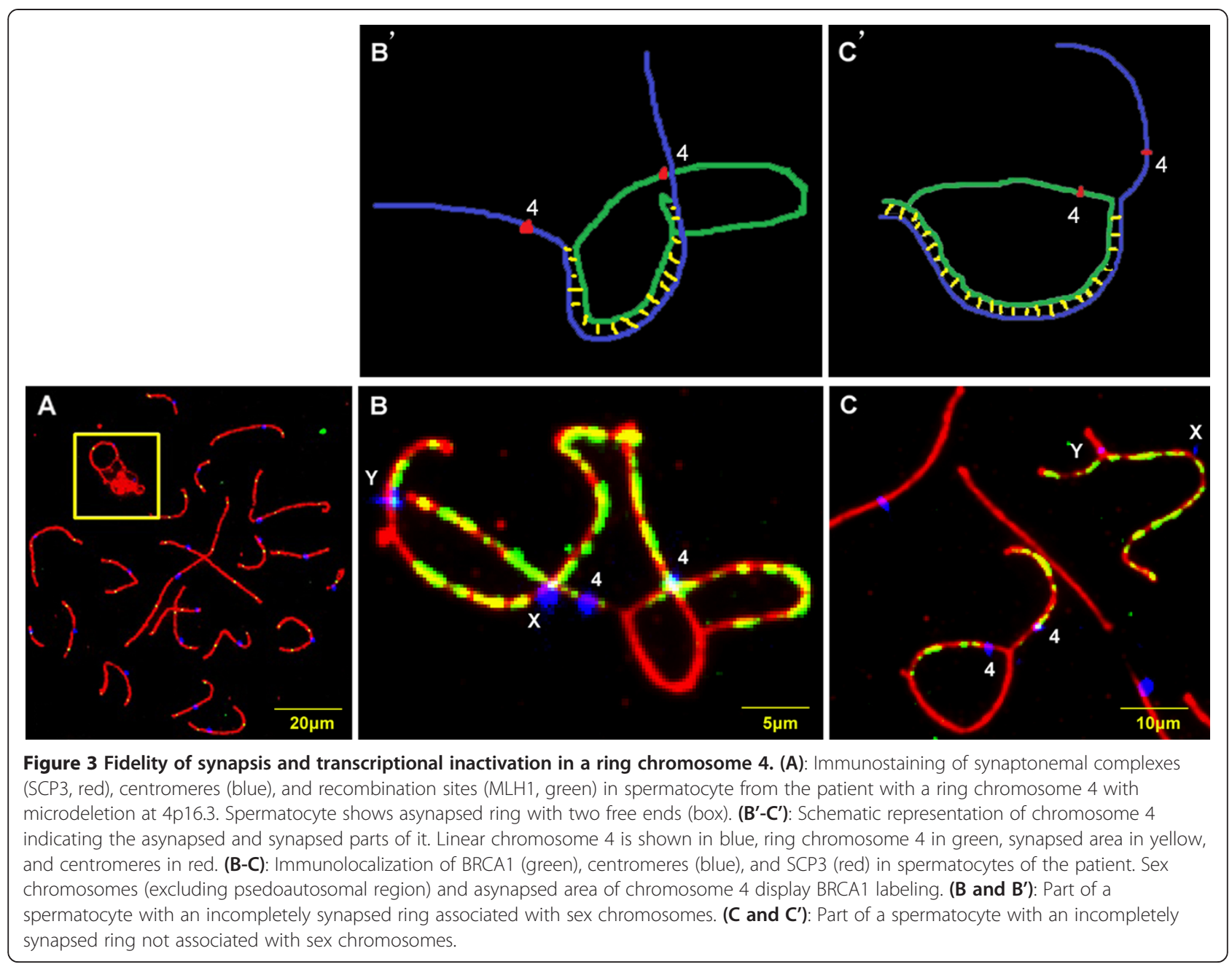

found in our patient, however, the mean MLH1 foci per cell number of our patient was still within the range of normal men $[25,26]$ shown in the previous literature. Thus, the decrease of MLH1 foci per cell in our patient might be due to interindividual variation, but not donor age and status [27]. Indeed, several reports described that differences in recombination could be explained by the presence of SNPs in genes involved in meiotic recombination. [28,29]. Therefore, it was difficult to assess the role of less MLH1 foci during the process of spermatogenesis in our patients.

It has been proposed that a low frequency of recombination in the psedoautosomal region may associate to infertility in azoospermic and severe oligospermic patients who did not carry a reorganization [26]. While there is no significant difference between our patient and the controls in terms of $\mathrm{XY}$ recombination frequency, which may support our hypothesis that the presence of the ring chromosome influences the meiosis of the patient.
The ring chromosome 4 had a $852 \mathrm{~kb}$ deletion at 4p16.3. No direct evidence from the literature indicates that the 17 deleted genes at $4 \mathrm{p} 16.3$ are associated with spermatogenesis. The functions of some the genes are still unknown. Therefore, the possibility that the 17 genes on $4 \mathrm{p} 16.3$ resulted in spermatogenic arrest could not be excluded.

In addition, some other potential factors may interfere with spermatogenesis for the patient, such as deleterious effect of left cryptorchidism [30], or presence of interchromosomal effect (ICE) [22] due to carrying a ring chromosome 4. However, no direct evidences in our experiments were found from the patient. The molecular mechanism should be further investigated.

\section{Conclusion}

In summary, we report a ring chromosome 4 with a $852 \mathrm{~kb}$ deletion at $4 \mathrm{p} 16.3$ that might affect the progression of meiosis I prophase, synapsis, and transcriptional activation of asynapsed areas, leading to spermatogenic 
arrest. Additional cases and future research will determine if a single deleted gene, a cluster of genes deleted at $4 \mathrm{p} 16.3$, or the chromosome structural abnormality contribute to impaired spermatogenesis.

\section{Competing interest}

The authors declare that they have no competing interests.

\section{Authors' contributions}

QY, LW and HLG played a role in biological experiments and molecular diagnosis. BY and YXC had major roles in patient and control recruitment, collection and analysis of clinical data. All authors contributed to the writing and revision of the manuscript.

\section{Acknowledgements}

We thank the patient and his parents for their cooperation in the study. This work was supported in part by the National Natural Science Foundation of China (30901652) and the Natural Science Foundation of Jiangsu Province (BK2010462, BK2011660)

\section{Author details}

${ }^{1}$ Institute of Laboratory Medicine, Jinling Hospital, Nanjing University School of Medicine, 305 East Zhongshan Road, Nanjing 210002, PR China. ${ }^{2}$ Institute of Reproductive Medicine, Jinling Hospital, Nanjing University School of Medicine, 305 East Zhongshan Road, Nanjing 210002, PR China. ${ }^{3}$ Laboratory of Molecular and Cell Genetics, Hefei National Laboratory for Physical Sciences at Microscale and School of Life Sciences, University of Science and Technology of China, Hefei, China.

Received: 24 March 2014 Accepted: 25 June 2014

Published: 1 July 2014

\section{References}

1. Kirkpatrick G, Chow V, Ma S: Meiotic recombination, synapsis, meiotic inactivation and sperm aneuploidy in a chromosome 1 inversion carrier. Reprod Biomed Online 2012, 24:91-100.

2. Ferguson KA, Chow V, Ma S: Silencing of unpaired meiotic chromosomes and altered recombination patterns in an azoospermic carrier of a $\mathrm{t}(8 ; 13)$ reciprocal translocation. Hum Reprod 2008, 23:988-995.

3. Leng M, Li G, Zhong L, Hou H, Yu D, Shi Q: Abnormal synapses and recombination in an azoospermic male carrier of a reciprocal translocation $\mathrm{t}(1 ; 21)$. Fertil Steril 2009, 91:1293.e17-22.

4. Coco R, Rahn MI, Estanga PG, Antonioli G, Solari AJ: A constitutional complex chromosome rearrangement involving meiotic arrest in an azoospermic male: case report. Hum Reprod 2004, 19:2784-2790.

5. Guilherme RS, Meloni VF, Kim CA, Pellegrino R, Takeno SS, Spinner NB, Conlin LK, Christofolini DM, Kulikowski LD, Melaragno MI: Mechanisms of ring chromosome formation, ring instability and clinical consequences. BMC Med Genet 2011, 12:171.

6. Mcllree ME, Price WH, Brown WM, Tulloch WS, Newsam JE, Maclean N: Chromosome studies on testicular cells from 50 subfertile men. Lancet 1966, 2:69-71

7. Moreau N, Teyssier M: Ring chromosome 15: report of a case in an infertile man. Clin Genet 1982, 21:272-279.

8. Huret $\mathrm{J}$, Leonard C, Kanoui V: Ring chromosome 21 in a phenotypically normal but infertile man. Clin Genet 1985, 28:541-545.

9. Dallapiccola B, De Filippis V, Notarangelo A, Perla G, Zelante L: Ring chromosome 21 in healthy persons: different consequences in females and in males. Hum Genet 1986, 73:218-220

10. Yoshida A, Miura K, Shirai M: Cytogenetic survey of 1,007 infertile males. Urol Int 1997, 58:166-176.

11. Slater HR, Nouri S, Earle E, Lo AW, Hale LG, Choo KH: Neocentromere formation in a stable ring 1p32-p36.1 chromosome. J Med Genet 1999, 36:914-918.

12. Stahl BC, Patil SR, Syrop CH, Sparks AE, Wald M: Supernumerary minute ring chromosome 14 in a man with primary infertility and left varicocele. Fertil Steril 2007, 87:1213.e1-3.

13. Martin JR, Wold A, Taylor HS: Ring chromosome 12 and severe oligospermia: a case report. Fertil Steril 2008, 90(443):e13-15.
14. Hammoud I, Gomes DM, Bergere M, Wainer R, Selva J, Vialard F: Sperm chromosome analysis of an infertile patient with a $95 \%$ mosaic $\mathrm{r}(21)$ karyotype and normal phenotype. Fertil Steril 2009, 91(930):e13-15.

15. Zuccarello D, Dallapiccola B, Novelli A, Foresta C: Azoospermia in a man with a constitutional ring 22 chromosome. Eur J Med Genet 2010, 53:389-391.

16. Rajesh H, Freckmann ML, Chapman M: Azoospermia and paternal autosomal ring chromosomes: case report and literature review. Reprod Biomed Online 2011, 23:466-470.

17. Judis L, Chan ER, Schwartz S, Seftel A, Hassold T: Meiosis I arrest and azoospermia in an infertile male explained by failure of formation of a component of the synaptonemal complex. Fertil Steril 2004, 81:205-209.

18. Concolino D, Rossi E, Strisciuglio P, lembo MA, Giorda R, Ciccone R, Tenconi R, Zuffardi O: Deletion of a $760 \mathrm{~kb}$ region at $4 \mathrm{p} 16$ determines the prenatal and postnatal growth retardation characteristic of Wolf-Hirschhorn syndrome. J Med Genet 2007, 44:647-650.

19. Soysal Y, Balci S, Hekimler K, Liehr T, Ewers E, Schoumans J, Bui TH, Içduygu FM, Kosyakova N, Imirzalioğlu N: haracterization of double ring chromosome 4 mosaicism associated with bilateral hip dislocation, cortical dysgenesis, and epilepsy. Am J Med Genet A 2009, 149A:2782-2787.

20. Misceo D, Barøy T, Helle JR, Braaten O, Fannemel M, Frengen E: 1.5Mb deletion of chromosome 4p16.3 associated with postnatal growth delay, psychomotor impairment, epilepsy, impulsive behavior and asynchronous skeletal development. Gene 2012, 50:85-91.

21. Manterola M, Page J, Vasco C, Berríos S, Parra MT, Viera A, Rufas JS, Zuccotti M, Garagna S, Fernández-Donoso R: A high incidence of meiotic silencing of unsynapsed chromatin is not associated with substantial pachytene loss in heterozygous male mice carrying multiple simple robertsonian translocations. PLoS Genet 2009, 5(8)::1000625.

22. Burgoyne PS, Mahadevaiah SK, Turner JM: The consequences of asynapsis for mammalian meiosis. Nat Rev Genet 2009, 10(3):207-216.

23. Wojtasz L, Cloutier JM, Baumann M, Daniel K, Varga J, Fu J, Anastassiadis K, Stewart AF, Reményi A, Turner JM, Tóth A: Meiotic DNA double-strand breaks and chromosome asynapsis in mice are monitored by distinct HORMAD2-independent and -dependent mechanisms. Genes Dev 2012, 26:958-973.

24. Jiang H, Wang L, Cui Y, Xu Z, Guo T, Cheng D, Xu P, Yu W, Shi Q: Meiotic chromosome behavior in a human male $t(8 ; 15)$ carrier. J Genet Genomic 2014, 41:177-185.

25. Sun F, Trpkov K, Rademaker A, Ko E, Martin RH: Variation in meiotic recombination frequencies among human males. Hum Genet 2005, 116:172-178.

26. Codina-Pascual M, Oliver-Bonet M, Navarro J, Campillo M, García F, Egozcue S, Abad C, Egozcue J, Benet J: Synapsis and meiotic recombination analyses: MLH1 focus in the XY pair as an indicator. Hum Reprod 2005, 20:2133-2139.

27. Lynn A, Koehler KE, Judis L, Chan ER, Cherry JP, Schwartz S, Seftel A, Hunt PA, Hassold TJ: Covariation of synaptonemal complex length and mammalian meiotic exchange rates. Science 2002, 296:2222-2225.

28. Chowdhury R, Bois PR, Feingold E, Sherman SL, Cheung VG: Genetic analysis of variation in human meiotic recombination. PLoS Genet 2009, 5(9):e1000648.

29. Reynolds A, Qiao H, Yang Y, Chen JK, Jackson N, Biswas K, Holloway JK, Baudat F, de Massy B, Wang J, Höög C, Cohen PE, Hunter N: RNF212 is a dosage-sensitive regulator of crossing-over during mammalian meiosis. Nat Genet 2013, 45:269-278.

30. Huff DS, Fenig DM, Canning DA, Carr MG, Zderic SA, Snyder HM 3rd: Abnormal germ cell development in cryptorchidism. Horm Res 2001, 55:11-17.

doi:10.1186/1755-8166-7-45

Cite this article as: Yao et al: Meiotic prophase I defects in an oligospermic man with Wolf-Hirschhorn syndrome with ring chromosome 4. Molecular Cytogenetics 2014 7:45. 